\title{
Factors associated with infection by Pseudomonas aeruginosa in adult cystic
} fibrosis

\author{
D. Chambers*, F. Scott", R. Bangur*, R. Davies*, A. Lim*, S. Walters ${ }^{\star}$, G. Smith ${ }^{+}$, \\ T. Pitt ${ }^{\#}$, D. Stableforth* and D. Honeybourne*
}

ABSTRACT: Cross-infection with Pseudomonas aeruginosa is an emerging issue in the care of patients with cystic fibrosis (CF). This study sought to determine the extent of, and patient factors associated with, cross-infection in a tertiary referral adult CF centre.

$P$. aeruginosa isolates were genotyped into two groups between November 2001 and February 2003, using pulsed-field gel electrophoresis after DNA digestion by the Spel endonuclease, and identified as clustered if there was $>\mathbf{8 0} \%$ homology in the macrorestriction profiles. Patient factors and measures of disease severity were identified a priori.

In total, 157 out of 227 patients had a $P$. aeruginosa isolate genotyped. Of these, 94 patients (60\%) were infected with clustered genotypes and $47(30 \%)$ were infected with the newly described "Midlands 1" (Md1) genotype. A further 18 patients were infected with the previously identified "Liverpool" genotype and two with the "Manchester" genotype. Logistic regression analysis revealed that the predominant predictor of infection with Md1 was age at the time of referral to the centre, suggesting that infection may have occurred prior to referral in some patients. Md1 demonstrated a relatively benign anti-biogram and did not appear to be associated with more severe disease.

In conclusion, the present study provides further evidence of the emerging importance of Pseudomonas aeruginosa cross-infection in cystic fibrosis.

\section{KEYWORDS: Cross-infection, cystic fibrosis, Pseudomonas aeruginosa}

$\mathbf{R}$ espiratory tract infection by the pathogen Pseudomonas aeruginosa in patients with cystic fibrosis (CF) is associated with progression of disease and increased mortality [1]. Although cross-infection with $P$. aeruginosa in siblings with CF is well documented [2], crossinfection in other settings has been thought to be rare. There is now increasing evidence of the importance of cross-infection in paediatric $[3,4]$ and, more recently, adult [4-7] CF centres. There is naturally concern among patients, families and carers that this spread may adversely impact upon morbidity and mortality.

The number of terms used in the literature to describe these cross-infecting $P$. aeruginosa isolates ("epidemic", "multiresistant", "transmissible") reflects the lack of knowledge about their epidemiology, microbiology and potential for increased pathogenicity. There is an urgent need for more data in these areas to guide decisions about the future of CF care.

The West Midlands Adult Cystic Fibrosis Centre (WMACFC; Birmingham, UK) is a large tertiary referral centre providing both outpatient and inpatient services to CF patients from a catchment area covering most of central England. This study was completed as part of a nationwide surveillance project [8] with three aims: 1) to assess the centre patient population for evidence of cross-infection by $P$. aeruginosa; 2) to identify patient factors associated with cross-infection; and 3) to determine if cross-infection is associated with more severe disease.

\section{METHODS}

Sputum or cough swab samples were obtained from patients registered with the WMACFC from November 2001 to February 2003. Samples were collected in two groups. The first group was collected during routine clinic visits from November 2001 to May 2002. Patients who did not have a $P$. aeruginosa isolate genotyped in the first group were approached again either at routine clinics or by post between December 2002 and February 2003. An attempt was made to include all patients known to be colonised with Pseudomonas spp. on the database.

\section{AFFILIATIONS}

*West Midlands Adult Cystic Fibrosis Centre,

+Dept of Microbiology, Birmingham Heartlands \& Solihull NHS Trust, and "Public Health Dept, University of Birmingham, Birmingham, and ${ }^{*}$ Laboratory of HealthCare Associated Infection, Health protection Agency, Colindale, London, UK.

\section{CORRESPONDENCE}

D. Honeybourne

West Midlands Adult Cystic Fibrosis Centre

Birmingham Heartlands \& Solihull NHS Trust

Bordesley Green East

Birmingham

B9 5SS

UK

Fax: 441214241661

E-mail: david.honeybourne@

heartofengland.nhs.uk

\section{Received:}

November 052004

Accepted after revision:

June 192005 
Sputum was obtained from patients at outpatient visits and occasionally by post or from inpatients. Specimens were processed using the standard operating procedure, which included a homogenisation step with the addition of an equal volume of sputasol. Mixing and vortexing were carried out prior to inoculation of culture plates. Each medium was inoculated directly onto blood agar with an optochin disc. Three selective media for $P$. aeruginosa, Burkholderia cepacia and Staphylococcus aureus and a 1:500 dilution in sterile distilled water were inoculated onto chocolated blood agar for semiquantitative analysis. All media were incubated for $48 \mathrm{~h}$ and B. cepacia selective agar was incubated for 1 week. Reading occurred at $48 \mathrm{~h}$ and 1 week. Organisms assessed as significant were selected for identification and susceptibility testing using British Society for Antimicrobial Chemotherapy (BSAC) methodology, including testing for colomycin. Gram-negative organisms that were resistant to colomycin or were isolated from the selective $B$. cepacia media were identified and sent to the reference laboratory for confirmation.

Samples underwent standard microbiological analysis, including culture on a range of selective media and susceptibility testing using disc diffusion, following procedures laid down by BSAC. Where $P$. aeruginosa was isolated, a sweep of colonies was taken from primary culture plates and submitted in a coded fashion to the Laboratory of HealthCare Associated Infection (London, UK) for inclusion in a cross-infection survey involving $31 \mathrm{CF}$ centres in England and Wales. Isolates were genotyped using pulsed-field gel electrophoresis following digestion of DNA by the SpeI endonuclease, as described previously [8]. Genotypes were identified as clustered if there was $>80 \%$ homology in their DNA macrorestrictrion profile.

Prior to obtaining sputum samples, patient factors felt to either be of potential importance in determining colonisation by a clustered $P$. aeruginosa genotype or to be related to disease severity were identified. The following information was obtained from case notes: 1) demographics; 2) CF genotype; 3) CF phenotype (pancreatic function, diabetes mellitus, CFrelated liver disease); 4) presence of allergic bronchopulmonary aspergillosis; 5) source of referral to the WMACFC; 6) age at the time of referral; 7) the number of months attending the WMACFC; 8) participation in shared care; 9) lung transplantation and Burkholderia cepacia infection status; 10) the number of hospital admissions; and 11) i.v. antibiotic courses for the previous 6 yrs. Patients were defined as being pancreatic insufficient if pancreatic enzyme supplements were currently prescribed by a consultant physician. The presence or absence of CF liver disease was noted from information obtained from case notes, specifically the findings on examination and the results of liver ultrasound scans. Body mass index was calculated and the forced expiratory volume in one second (FEV1) was assessed at the time of sputum collection using the same dry bellows spirometer and disposable, valved mouthpieces (Vitalograph, Buckingham, UK) calibrated daily (except for B. cepacia-infected patients where an identical model was used).

Comparisons were made between patients infected with the major clustered $P$. aeruginosa genotypes and patients infected with their own unique genotype. The patients' residential postcode was plotted and visually inspected for geographic clustering. Analysis was carried out using Chi-squared tests for categorical variables and unpaired t-tests or ANOVA for continuous variables. The Bonferroni correction was included when multiple comparisons were made. The Levene statistic was calculated to confirm homogeneity of variances between groups. Where variances were not homogeneous the KruskalWallis test was used. The study was approved by the local research ethics committee (Birmingham, UK).

\section{RESULTS}

At the time the study was conducted there were 227 patients attending the WMACFC, of whom $182(80 \%)$ were known to be colonised with $P$. aeruginosa. In total, 157 patients (86\% of those colonised) had a $P$. aeruginosa isolate genotyped, 113 patients in the first group and a further 44 in the second group. An attempt was made to include all patients known to be colonised with Pseudomonasaeruginosa on the database. However, during the study period, 25 patients were unable or unwilling to attend and provide samples. These were often patients who had been poor attendees at clinics for several years. Otherwise, there were no apparent differences with those captured in the study.

In total, 47 patients $(30 \%)$ were found to be infected with the previously unidentified clustered $P$. aeruginosa genotype Midlands 1 (Md1) [8]. Seven patients were infected with $B$. cepacia, of whom three were co-infected with the P. aeruginosa Md1 genotype. A further 18 patients (11.5\%) were infected with a previously described clustered $P$. aeruginosa genotype first identified in the Liverpool paediatric CF centre (the Liverpool genotype) [3]. Two patients were infected with a clustered genotype previously identified in patients attending an adult CF centre in Manchester (the Manchester genotype) [5]. None of these patients had ever attended clinics in Manchester or Liverpool. A further 27 patients (17\%) were infected with eight other small, previously unidentified, clusters (arbitrarily named C, D, E, F, H, I, J and K). Only 63 patients $(40 \%)$ were infected with their own unique $P$. aeruginosa genotype. These findings are summarised in table 1.

\begin{tabular}{lcc}
\hline TABLE 1 & $\begin{array}{c}\text { Clustered Pseudomonas aeruginosa isolates at } \\
\text { the West Midlands Adult Cystic Fibrosis Centre }\end{array}$ \\
\hline Cluster & Patients n & Total population \% \\
\hline Md1 & 47 & 30 \\
Liverpool & 18 & 11.5 \\
Manchester & 2 & \\
C & 4 & \\
D & 3 & 18.5 \\
E & 2 & \\
F & 4 & \\
H & 2 & \\
I & 2 & \\
J & 7 & \\
K & 3 & \\
Unique & 63 & \\
Total & 157 & \\
\hline & & \\
Md1: Midlands 1. & &
\end{tabular}


In total, 23 patients had $P$. aeruginosa isolates genotyped in both groups. Of these patients, three $(13 \%)$ had a different genotype identified in the second group (two patients initially had a Liverpool genotype isolated and then a unique genotype, and one patient initially $\mathrm{J}$ and then unique). In these instances the patient was deemed to have been infected with the clustered genotype for the purposes of the subsequent analysis.

There was no evidence of geographic clustering of clustered genotypes across the $\mathrm{CF}$ centre catchment area. The patient characteristics for each infecting $P$. aeruginosa genotype are presented in table 2 . On univariate analysis, patients infected with the Md1 genotype were younger, had been referred to the centre at a younger age, and were more likely to have been referred from the regional paediatric $\mathrm{CF}$ centre than those infected with a unique genotype. Patients infected with the Liverpool genotype were also younger and had been referred at a younger age. There were no significant differences between the patient groups for the other parameters assessed. Specifically, the infecting $P$. aeruginosa genotype did not appear to be associated with FEV1 (\% predicted), body mass index, the number of hospital admissions or the number of $i . v$. antibiotic courses prescribed, although these parameters tended to be worse in patients infected with the Liverpool genotype. Logistic regression analysis revealed that age at the time of referral to the centre was the predominant predictor of infection with the Md1 genotype. Further analysis revealed that patients referred from the regional paediatric centre $(n=96)$ were, as would be expected, significantly younger at the time of referral than those referred from elsewhere $(n=60$; 17.5 versus 21.9 yrs; $\mathrm{p}<0.001$ ).
The antibiotic resistance patterns of the clustered $P$. aeruginosa isolates varied considerably (table 3 ). The Liverpool genotype was generally more resistant, particularly to ciprofloxacin, the aminoglycosides and aztreonam, but there was a notable complete absence of resistance to piperacillin and colomycin in this group. Md1 genotypes were notably more resistant to colomycin, but otherwise resistance was similar to the unique $P$. aeruginosa genotypes. Generally, however, the anti-biogram was of little value in terms of predicting $P$. aeruginosa genotype.

\begin{tabular}{|c|c|c|c|c|c|}
\hline \multirow[t]{2}{*}{ TABLE 3} & \multicolumn{5}{|c|}{$\begin{array}{l}\text { Percentage of isolates resistant or intermediately } \\
\text { resistant to anti-pseudomonal antibiotics by } \\
\text { genotype }\end{array}$} \\
\hline & Unique & Md1 & Liverpool & Other & Total \\
\hline Ciprofloxacin & $25^{\star}$ & 32 & 52 & 24 & 30 \\
\hline Gentamicin & $33^{\star}$ & 36 & 61 & 32 & 37 \\
\hline Tobramycin & $6^{*}$ & 10 & 42 & 3 & 11 \\
\hline Amikacin & $26^{*}$ & 17 & 48 & 11 & 24 \\
\hline Ceftazidime & 8 & 10 & 19 & 5 & 10 \\
\hline Aztreonam & $19^{*}$ & 8 & 42 & 8 & 17 \\
\hline Colomycin & $3^{*}$ & 13 & 0 & 8 & 6 \\
\hline Piperacillin & 9 & 6 & 0 & 3 & 6 \\
\hline Piperacillin/tazobactum & 5 & 5 & 0 & 0 & 4 \\
\hline
\end{tabular}

Data presented as a percentage. Md1: Midlands 1. *: $p<0.05$ for unique versus Md1 versus Liverpool.

TABLE 2 Patient characteristics by infecting Pseudomonas aeruginosa genotype

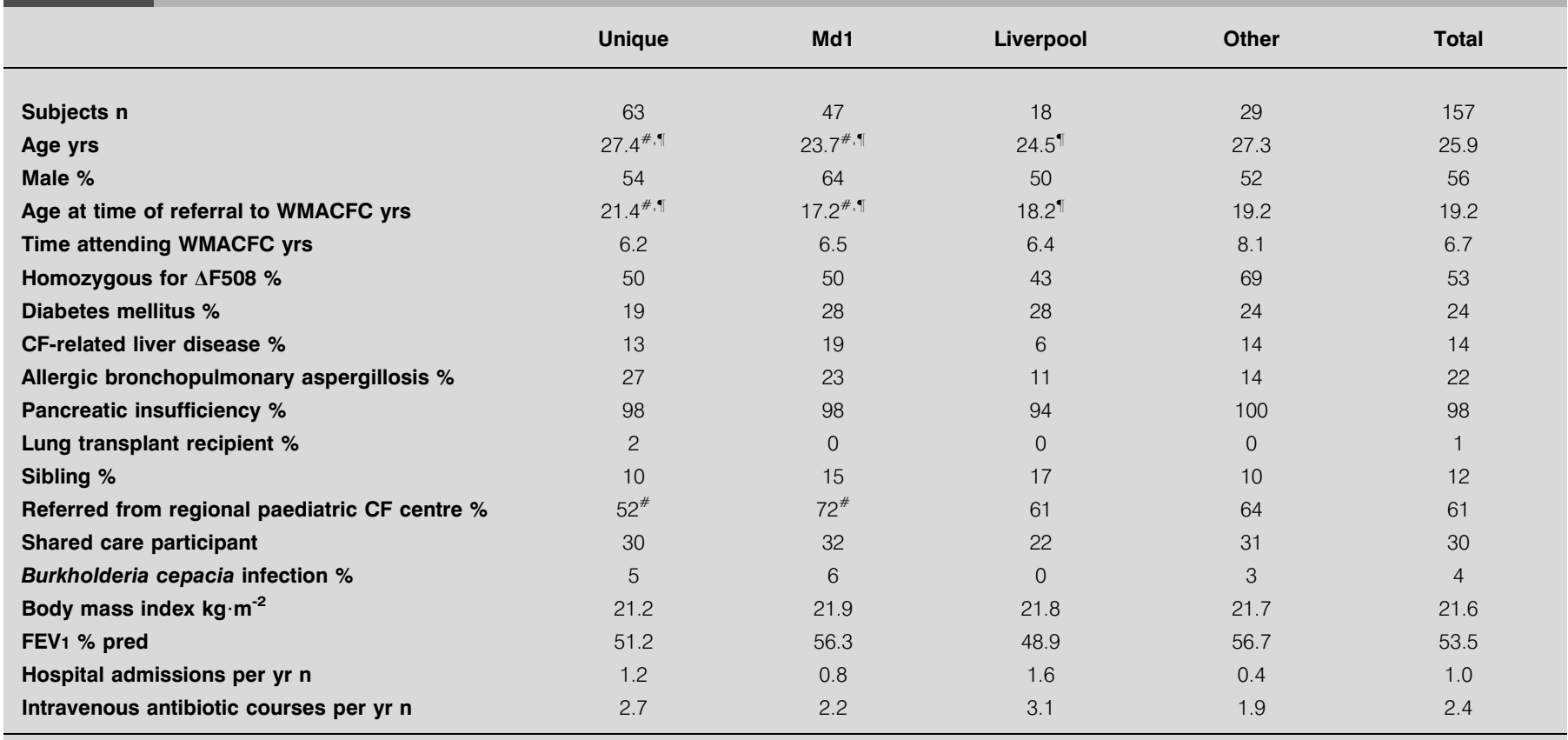

Data presented as mean unless otherwise stated. Md1: Midlands 1; WMACFC: West Midlands Adult Cystic Fibrosis Centre; CF: cystic fibrosis; FEV1: forced expiratory volume in one second. ${ }^{*}: p<0.05$ for unique versus Md1; $"$ : $p<0.05$ for unique versus Md1 versus Liverpool. 


\section{DISCUSSION}

This study demonstrates considerable clustering of $P$. aeruginosa isolates obtained from a population of $157 \mathrm{CF}$ patients attending a CF centre. In total, $60 \%$ of patients were infected with clustered genotypes and half of these were infected by a common genotype (Md1). Of these, 18 patients were found to be infected with the previously described Liverpool genotype [3]. None of these patients had ever attended the CF centre in Liverpool, however, a few (table 2) had attended the regional CF-related liver disease clinic (a clinic attended by patients from both the WMACFC and Liverpool CF centre). A further two patients were infected with the previously identified Manchester genotype. Although environmental sources cannot be entirely excluded, the clustered $P$. aeruginosa isolates identified in this study are likely to have been acquired through cross-infection. A number of studies that have identified clustered $P$. aeruginosa isolates in patients with CF have attempted to identify environmental sources without success $[3,6,9]$. However it is possible that similar genetic fingerprints for organisms may originate from diverse sources. Social contact may, therefore, not be the main problem.

However, multiple reports from the UK, Australia and Norway suggest that cross-infection between unrelated patients occurs much more frequently than previously recognised [3-7, 10]. In keeping with the current findings, the studies from Liverpool, Melbourne, Brisbane and Bergen all found that the majority of $P$. aeruginosa-infected patients attending their centres harboured clonal isolates. Typically, most patients are infected with a single clonal genotype (e.g. Liverpool or Md1) while the remainder are infected with a number of other genotypes $[3,4,6,10]$. The findings of the Brisbane group in particular very closely mirror the current findings, as $59 \%$ of patients were infected with a clonal genotype (39\% with the same genotype (pulsotype 2 ) and $20 \%$ with multiple other small clusters) [4].

Patients infected with the Md1 genotype were younger than patients infected with unique genotypes, had been referred to the centre at a younger age and were more likely to have been referred from the regional paediatric CF centre (table 2). However, logistic regression analysis suggested that the major factor determining the infecting $P$. aeruginosa genotype was age at the time of referral to the centre. It is possible that the age at the time of referral simply relates to source of referral, given that patients referred from the regional paediatric CF centre are younger at the time of referral than patients referred from elsewhere (17.5 versus 21.9 yrs; $\mathrm{p}<0.0001)$. It is probable that some patients were already infected with the Md1 genotype at the time of referral from the paediatric centre. In support of this suggestion is the data provided by the strictly segregated $B$. cepacia infected cohort. Two of the three patients with $B$. cepacia and Md1 P. aeruginosa co-infection (both of whom were referred from the regional paediatric CF centre) have had little or no contact with other patients at the adult centre due to a strict segregation policy. This suggests that Md1 P. aeruginosa may have been acquired at the paediatric centre. These findings are in line with those of the Brisbane group who demonstrated that patients attending the regional paediatric CF centre were infected with their predominant clonal isolate (pulsotype 2) and that adult patients infected with pulsotype 2 were younger and more likely to have been referred from the paediatric centre [4].

Overall it would appear that infection with the more resistant Liverpool genotype at the WMACFC (table 3) may be associated with more severe disease, since the mean number of hospital admissions and courses of i.v. antibiotics required were higher in this group of patients and the FEV1 lower, although individually the differences were not statistically significant (table 2). Contemporary UK colomycin resistance rates are $\sim 3 \%$. There is not enough accurate information to comment on whether patients with Md1 strains were more likely to have been treated with colomycin in the past.

Patients infected with the clustered genotype in Melbourne were more likely to be using recombinant DNAse, more likely to be hospitalised and had poorer FEV1 [6]. Patients in Brisbane suspected of cross-infection also had a poorer FEV1, while patients infected with the clustered genotype in Manchester received more i.v. antibiotics and had more hospital admissions than patients infected with unique genotypes [11]. Recently, the Sheffield group has reported similar adverse features in patients infected with their local clonal strain [7]. In contrast, infection with the Md1 genotype did not appear to be associated with more severe disease in terms of FEV1, i.v. antibiotic use or number of hospitalisations. A similarly benign $P$. aeruginosa genotype has been identified in Norway [10]. This finding is important since it suggests that, at least for these $P$. aeruginosa genotypes, the bacterial factors associated with the ability to more successfully establish persistent infection may not necessarily be the same as those associated with virulence and severe disease, as had originally been feared [3, 11].

Generally, clustered $P$. aeruginosa genotypes have been found to be more resistant to anti-pseudomonal antibiotics $[3,5,6]$. The Md1 genotype, in contrast, displayed a relatively benign anti-biogram, except for a notably higher prevalence of resistance to colomycin (table 3). Overall the anti-biogram was of little practical value in identifying clustered strains in individual cases.

Some limitations of this study should be recognised. Since only a sweep of colonies of $P$. aeruginosa and not individual colonies from a variety of morphotypes was sampled from each culture plate, the study did not examine whether patients were colonised with more than one genotype. In most previous studies, however, patients maintain a single genotype for many years, although it may display dramatically varied morphotypes on the culture plate [12-18]. Another limitation is that the present study involves only a single cross-section of the clinic population. Further longitudinal studies will be required to confirm the stability of the infecting clustered and unique $P$. aeruginosa genotypes over time. Longitudinal data, rather than the cross-sectional data presented here, are also required before firm conclusions about disease progression in the patients infected with clustered genotypes can be drawn.

The bacterial factors leading one $P$. aeruginosa strain to set up chronic infection or supplant another chronically infecting strain in a patient while other genotypes colonise or infect only briefly before moving on are unknown. Few studies have examined this question. The Manchester genotype does not appear to induce an exaggerated inflammatory response in its 
host despite increased treatment requirements in these patients [19]. In an Australian study, eight out of 18 patients were found to be infected with a clustered $P$. aeruginosa genotype, and this strain lacked the functional mucA mutations responsible for over-production of alginate and mucoidy in the unique strains [20]. In other words, alternative mechanisms must have been operational in the clustered strain to lead to mucoidy [20]. Multiple and diverse bacterial factors associated with the ability of cross-infecting $P$. aeruginosa strains to successfully establish persistent infection in other hosts are conceivable including: an improved ability to aerosolise [9]; a propensity to infect sheltered extrapulmonary sites [21] or adhere to CF mucosal surfaces [22]; a better ability to prosper in the abnormally anaerobic environment of the airway surface liquid typical of older CF patients; an increased tendency to form biofilms in the respiratory tract; or increased intrinsic antibiotic resistance [3] (in turn related to the propensity to form biofilms) [23]. Further work is required to better define these bacterium-specific factors.

The present study demonstrates that clustered genotypes are not necessarily associated with a poorer prognosis. It is becoming apparent that these strains become widespread not through gross breakdowns in infection control (although clearly cross-infection remains the major means of acquisition), but more importantly through changes specific to the bacterium. These changes, although yet to be defined, improve the strain's ability to supplant previously infecting strains and/or establish persistent infection. Elucidation of these bacteria-specific factors may provide information which will be of wider benefit to the CF community.

In view of the current findings, patients were sent a letter emphasising the importance of minimising patient-to-patient contact for outpatients as well as inpatients. Fortunately, a new inpatient facility with 20 single rooms and an outpatient clinic with nine outpatient rooms was opened in November 2002. These changes enabled a more efficient process of patient segregation to occur. The current authors believe that this new arrangement will considerably reduce the risk of cross infection between patients, and plan to continue surveillance of $P$. aeruginosa genotypes in the future to assess the impact of these environmental changes.

In conclusion, clustered strains of Pseudomonas aeruginosa (predominantly of Midlands 1 genotype) were found in $60 \%$ of patients attending the West Midlands Adult Cystic Fibrosis Centre, with some patients being infected with genotypes more commonly associated with geographically distant centres. Infection with the Midlands 1 genotype, found in 30\% of those tested, did not appear to be associated with more severe disease. Patient factors were found to be of relatively minor importance in predicting infection with the Midlands 1 genotype, although the association with age at the time of referral to the centre suggests that infection may have been acquired in the relatively distant past in many patients.

\section{ACKNOWLEDGEMENTS}

The authors would like to thank the UK Cystic Fibrosis Trust for their assistance in carrying out the genotyping of P. aeruginosa isolates. The authors would also like to thank their physiotherapy colleagues for their help in obtaining sputum samples.

\section{REFERENCES}

1 Pamukcu A, Bush A, Buchdahl R. Effects of Pseudomonas aeruginosa colonization on lung function and anthropometric variables in children with cystic fibrosis. Pediatr Pulmonol 1995; 19: 10-15.

2 Grothues D, Koopmann U, von der Hardt H, Tummler B. Genome fingerprinting of Pseudomonas aeruginosa indicates colonization of cystic fibrosis siblings with closely related strains. J Clin Microbiol 1988; 26: 1973-1977.

3 Cheng K, Smyth RL, Govan JR, et al. Spread of beta-lactamresistant Pseudomonas aeruginosa in a cystic fibrosis clinic. Lancet 1996; 348: 639-642.

4 O'Carroll MR, Syrmis MW, Wainwright CE, et al. Clonal strains of Pseudomonas aeruginosa in paediatric and adult cystic fibrosis units. Eur Respir J 2004; 24: 101-106.

5 Jones AM, Govan JR, Doherty CJ, et al. Spread of a multiresistant strain of Pseudomonas aeruginosa in an adult cystic fibrosis clinic. Lancet 2001; 358: 557-558.

6 Armstrong DS, Nixon GM, Carzino R, et al. Detection of a widespread clone of Pseudomonas aeruginosa in a pediatric cystic fibrosis clinic. Am J Respir Crit Care Med 2002; 166: 983-987.

7 Edenborough FP, Stone HR, Kelly SJ, Zadik P, Doherty CJ, Govan JRW. Genotyping of Pseudomonas aeruginosa in cystic fibrosis suggests need for segregation. J Cyst Fibros 2004; 3: 37-44.

8 Scott FW, Pitt TL. Identification and characterization of transmissible Pseudomonas aeruginosa strains in cystic fibrosis patients in England and Wales. J Med Microbiol 2004; 53: 1-7.

9 Jones AM, Govan JR, Doherty CJ, et al. Identification of airborne dissemination of epidemic multiresistant strains of Pseudomonas aeruginosa at a CF centre during a cross infection outbreak. Thorax 2003; 58: 525-27.

10 Fluge G, Ojeniyi B, Hoiby N, et al. Typing of Pseudomonas aeruginosa strains in Norwegian cystic fibrosis patients. Clin Microbiol Infect 2001; 7: 238-243.

11 Jones AM, Dodd ME, Doherty CJ, Govan JR, Webb AK. Increased treatment requirements of patients with cystic fibrosis who harbour a highly transmissible strain of Pseudomonas aeruginosa. Thorax 2002; 57: 924-925.

12 Renders NH, Sijmons MA, van Belkum A, Overbeek SE, Mouton JW, Verbrugh HA. Exchange of Pseudomonas aeruginosa strains among cystic fibrosis siblings. Res Microbiol 1997; 148: 447-454.

13 Romling U, Fiedler B, Bosshammer J, et al. Epidemiology of chronic Pseudomonas aeruginosa infections in cystic fibrosis. J Infect Dis 1994; 170: 1616-1621.

14 Struelens MJ, Schwam V, Deplano A, Baran D. Genome macrorestriction analysis of diversity and variability of Pseudomonas aeruginosa strains infecting cystic fibrosis patients. J Clin Microbiol 1993; 31: 2320-2326.

15 Fegan M, Francis P, Hayward AC, Fuerst JA. Heterogeneity, persistence, and distribution of Pseudomonas aeruginosa genotypes in cystic fibrosis patients. J Clin Microbiol 1991; 29: 2151-2157.

16 Aaron SD, Ramotar K, Ferris W, et al. Adult cystic fibrosis exacerbations and new strains of Pseudomonas aeruginosa. Am J Respir Crit Care Med 2004; 169: 811-815.

17 Breitenstein S, Walter S, Bosshammer J, Romling U, Tummler B. Direct sputum analysis of Pseudomonas 
aeruginosa macrorestriction fragment genotypes in patients with cystic fibrosis. Med Microbiol Immunol (Berl) 1997; 186: 93-99.

18 Wolz C, Kiosz G, Ogle JW, et al. Pseudomonas aeruginosa crosscolonization and persistence in patients with cystic fibrosis. Use of a DNA probe. Epidemiol Infect 1989; 102: 205-214.

19 Jones AM, Martin L, Bright-Thomas RJ, et al. Inflammatory markers in cystic fibrosis patients with transmissible Pseudomonas aeruginosa. Eur Respir J 2003; 22: 503-506.

20 Anthony M, Rose B, Pegler MB, et al. Genetic analysis of Pseudomonas aeruginosa isolates from the sputa of Australian adult cystic fibrosis patients. J Clin Microbiol 2002; 40: 2772-2778.
21 Taylor RF, Morgan DW, Nicholson PS, Mackay IS, Hodson ME, Pitt TL. Extrapulmonary sites of Pseudomonas aeruginosa in adults with cystic fibrosis. Thorax 1992; 47: 426-428.

22 Tsang KW, Shum DK, Chan S, et al. Pseudomonas aeruginosa adherence to human basement membrane collagen in vitro. Eur Respir J 2003; 21: 932-938.

23 Aaron SD, Ferris W, Ramotar K, Vandemheen K, Chan F, Saginur R. Single and combination antibiotic susceptibilities of planktonic, adherent, and biofilm-grown Pseudomonas aeruginosa isolates cultured from sputa of adults with cystic fibrosis. J Clin Microbiol 2002; 40: 4172-4179. 